\title{
ALGEBRAIC DETERMINATION OF THE SECOND FUNDAMENTAL FORM OF A SURFACE BY ITS MEAN CURVATURE
}

\author{
T. Y. THOMAS
}

1. Introduction. The main purpose of the following paper is to show that in general the mean curvature of a surface yields an algebraic determination of its second fundamental form. We do this by deriving the explicit equations giving this determination. The continuity and differentiability properties of the various functions entering into the discussion will be assumed without special mention since these requirements are obvious from the methods and equations employed.

We denote the mean curvature by $H$ and the Gaussian curvature by $K$. The symbols $g_{\alpha \beta}$ and $b_{\alpha \beta}$ will be used to denote the symmetric components of the first and second fundamental forms of the surface (two-dimensional surface in Euclidean three space). Between these quantities we have the relations

$$
H=g^{\alpha \beta} b_{\alpha \beta} / 2 \text { and }\left|g_{\alpha \beta}\right| K=\left|b_{\alpha \beta}\right|=b_{11} b_{22}-b_{12}^{2} \text {, }
$$

where $\left|g_{\alpha \beta}\right|$ and $\left|b_{\alpha \beta}\right|$ stand for determinants. The first of these relations can be regarded as defining the mean curvature. The second is known as the Gauss equation. We may mention here also the Codazzi equations which play an important role in the following, that is, the equations $b_{\alpha \beta, \gamma}=b_{\alpha \gamma, \beta}$, where the "comma" denotes covariant differentiation based on the first fundamental form of the surface.

We shall find that the combination $H^{2}-K$ enters into most of the following equations. This quantity satisfies the condition $H^{2}-K \geqq 0$. For, if we choose a coordinate system such that at a point $P$ we have $g_{\alpha \beta}=\delta_{\alpha \beta}$, then at this point $2 H=b_{11}+b_{22}$ and hence

$$
\begin{aligned}
4\left(H^{2}-K\right) & =\left(b_{11}^{2}+2 b_{11} b_{22}+b_{22}^{2}\right)-4\left(b_{11} b_{22}-b_{12}^{2}\right) \\
& =\left(b_{11}^{2}-2 b_{11} b_{22}+b_{22}^{2}\right)+4 b_{12}^{2} \\
& =\left(b_{11}-b_{22}\right)^{2}+4 b_{12}^{2} \geqq 0 .
\end{aligned}
$$

Suppose that $H^{2}-K=0$ in a region $R$ of the surface. Then at a point $P$ of $R$ and relative to a coordinate system for which $g_{\alpha \beta}=\delta_{\alpha \beta}$ at $P$ we have

$$
b_{11}+b_{22}=2 H \text { and } \quad b_{11} b_{22}-b_{12}^{2}=H^{2} .
$$

Received by the editors December 14, 1944. 
Squaring the first of these equations and combining with the second leads to the result $\left(b_{11}-b_{22}\right)^{2}=0$. Hence $b_{11}=b_{22}$ at $P$. The first equation (1.2) then gives $b_{11}=H$ and $b_{22}=H$. From this and the second equation (1.2) we now have $b_{12}=0$. From these relations and the fact that $g_{\alpha \beta}=\delta_{\alpha \beta}$ we can now unite $b_{\alpha \beta}=H g_{\alpha \beta}$. These latter relations are independent of the coordinate system employed and hold at all points of the region $R$ in which the condition $H^{2}-K=0$ is satisfied. By differentiating both members of the relations $b_{\alpha \beta}=H g_{\alpha \beta}$ covariantly and then making use of the Codazzi equations we now easily deduce that $H=$ const. From $H^{2}-K=0$ it then follows that $K=$ const. Hence the condition $H^{2}-K=0$ in a region $R$ of a surface implies that the mean curvature $H$ and the Gaussian curvature $K$ are constant in $R$. We shall make use of this result in the following discussion.

2. Determination of the covariant derivative of the $b$ tensor. Differentiate each of the equations (1.1) covariantly to obtain

$$
\begin{aligned}
H,,_{1} & =\left[g^{11} b_{11,1}+2 g^{12} b_{12,1}+g^{22} b_{22,1}\right] / 2 \\
H, 2 & =\left[g^{11} b_{11,2}+2 g^{12} b_{12,2}+g^{22} b_{22,2}\right] / 2, \\
\left|g_{\alpha \beta}\right| K,_{1} & =b_{11,1} b_{22}+b_{11} b_{22,1}-2 b_{12} b_{12,1}, \\
\left|g_{\alpha \beta}\right| K,_{2} & =b_{11,2} b_{22}+b_{11} b_{22,2}-2 b_{12} b_{12,2} .
\end{aligned}
$$

Now make the substitutions

$$
g^{11}=g_{22} /\left|g_{\alpha \beta}\right|, \quad g^{12}=-g_{12} /\left|g_{\alpha \beta}\right|, \quad g^{22}=g_{11} /\left|g_{\alpha \beta}\right|
$$

in the first two of the above equations and then write the resulting equations in the form

$$
\begin{aligned}
& b_{11,1}=\left(1 / g_{22}\right)\left[2\left|g_{\alpha \beta}\right| H,_{1}+2 g_{12} b_{12,1}-g_{11} b_{22,1}\right], \\
& b_{22,2}=\left(1 / g_{11}\right)\left[2\left|g_{\alpha \beta}\right| H,_{2}+2 g_{12} b_{12,2}-g_{22} b_{11,2}\right] .
\end{aligned}
$$

Use these latter equations to eliminate the quantities $b_{11,1}$ and $b_{22,2}$ from the right members of the above equations for $K,_{1}$ and $K, 2$. Then making use of the Codazzi equations we find that

$$
\begin{aligned}
& 2\left(g_{12} b_{22}-g_{22} b_{12}\right) b_{11,2}+\left(g_{22} b_{11}-g_{11} b_{22}\right) b_{22,1} \\
& \quad=\left|g_{\alpha \beta}\right| g_{22} K,,_{1}-2\left|g_{\alpha \beta}\right| b_{22} H, 1 \\
& \left(g_{11} b_{22}-g_{22} b_{11}\right) b_{11,2}+2\left(g_{12} b_{11}-g_{11} b_{12}\right) b_{22,1} \\
& =\left|g_{\alpha \beta}\right| g_{11} K,,_{2}-2\left|g_{\alpha \beta}\right| b_{11} H, 2 .
\end{aligned}
$$

We consider these equations as equations to determine the quantities $b_{11,2}$ and $b_{22,1}$. The determinant of this system is 


$$
\Delta=\left|\begin{array}{rr}
2\left(g_{12} b_{22}-g_{22} b_{12}\right) & \left(g_{22} b_{11}-g_{11} b_{22}\right) \\
\left(b_{11} g_{22}-g_{22} b_{11}\right) & 2\left(g_{12} b_{11}-g_{11} b_{12}\right)
\end{array}\right| .
$$

When this determinant is expanded and the terms collected we find that $\Delta=4\left[H^{2}-K\right]\left|g_{\alpha \beta}\right|^{2}$. The solution by Cramer's rule gives

$$
\begin{array}{r}
b_{11,2}=\left(\left|g_{\alpha \beta}\right| / \Delta\right)\left[2 g_{12} g_{22} b_{11} K, 1-2 g_{11} g_{22} b_{12} K, 1-4 g_{12} b_{11} b_{22} H, 1\right. \\
+4 g_{11} b_{12} b_{22} H, g_{11}-g_{11} g_{22} b_{11} K, 2+g_{11}^{2} b_{22} K, 2 \\
\left.+2 g_{22} b_{11}^{2} H, 2-2 g_{11} b_{11} b_{22} H,,_{, 2}\right], \\
b_{22,1}=\left(\left|g_{\alpha \beta}\right| / \Delta\right)\left[g_{11} g_{12} b_{22} K, 2-2 g_{12} b_{11} b_{22} H, 2-2 g_{11} g_{22} b_{12} K, 2\right. \\
+4 g_{22} b_{11} b_{12} H, 2-g_{11} g_{22} b_{22} K,,_{1}+2 g_{11} b_{22}^{2} H, 1 \\
\left.+g_{22}^{2} b_{11} K, 1-2 g_{22} b_{11} b_{22} H\right] .
\end{array}
$$

Since in the right members of (2.1) we have $b_{12,1}=b_{11,2}$ and $b_{12,2}=b_{22,1}$, the equations (2.1), (2.2) and (2.3) give the complete determination of the quantities $b_{\alpha \beta, \gamma}$. If we consider these equations at an arbitrary point $P$ but relative to a coordinate system such that $g_{\alpha \beta}=\delta_{\alpha \beta}$ at $P$ they will be found to simplify considerably. Thus we have

$$
\begin{aligned}
b_{11,1}= & 2 H, 1-b_{22,1}, \quad b_{22,2}=2 H, 2-b_{11,2} \\
b_{11,2}= & \frac{\left(H^{2}-K\right)^{-1}}{4}\left[-2 b_{12} K_{, 1}+4 b_{12} b_{22} H_{, 1}-b_{11} K_{, 2}+b_{22} K_{, 2}\right. \\
& \left.+2 b_{11}^{2} H, 2-2 b_{11} b_{22} H, 2\right] \\
b_{22,1}= & \frac{\left(H^{2}-K\right)^{-1}}{4}\left[-2 b_{12} K_{, 2}+4 b_{11} b_{12} H_{, 2}-b_{22} K_{, 1}\right. \\
& \left.+2 b_{22}^{2} H,_{1}+b_{11} K_{, 1}-2 b_{11} b_{22} H\right]
\end{aligned}
$$

at a point $P$ where $g_{\alpha \beta}=\delta_{\alpha \beta}$. The quantities $b_{11,2}$ and $b_{22,1}$ in the right members of the first two equations (2.4) are considered to have the determination given by the last two of these equations.

3. Integrability conditions. We now deduce the integrability conditions derivable from the system (2.4). In conformity with the way in which the third and fourth equations of this system have been written we assume that $H^{2}-K>0$. Later we shall show that the final integrability relations are independent of this condition.

Since the covariant derivative of the fundamental tensor (components $g_{\alpha \beta}$ ) vanishes, it is permissible to differentiate the equations of the system (2.4) covariantly, the result of such differentiation giving relations which are valid at a point $P$ for which $g_{\alpha \beta}=\delta_{\alpha \beta}$. Thus let 
us differentiate the first equation (2.4) covariantly with respect to $x^{2}$ and then subtract the quantity $b_{11,2,1}$ from both members of the resulting equation. This gives

$$
b_{11,1,2}-b_{11,2,1}=2 H_{1,2}-b_{22,1,2}-b_{11,2,1} .
$$

The quantities $b_{11,2,1}$ and $b_{22,1,2}$ in the right member of (3.1) can be determined by covariant differentiation of the third and fourth equations of (2.4) respectively. For the difference appearing in the left member of (3.1) we have by Bianchi's identity

$$
\begin{aligned}
b_{11,1,2}-b_{11,2,1} & =-b_{\sigma 1} B_{112}^{\sigma}-b_{1 \sigma} B_{112}^{\sigma}=-2 b_{1 \sigma} B_{112}^{\sigma} \\
& =-2 b_{1 \sigma} g^{\sigma \tau} B_{\tau 112}=-2 b_{1 \sigma} g^{\sigma \tau}\left(g_{\tau 2} g_{11}-g_{\tau 1} g_{12}\right) K \\
& =-2 b_{12} g_{11} K+2 b_{11} g_{12} K=-2 b_{12} K,
\end{aligned}
$$

where the $B$ 's are the components of the curvature tensor and use has been made of the fact that $g_{\alpha \beta}=\delta_{\alpha \beta}$ in obtaining the final expression. When these substitutions are made in (3.1) we obtain a rather complicated equation which is capable of simplification. In connection with this process of simplification let us define the quantities

$$
b^{\alpha \beta}=B^{\alpha \beta} /\left|g_{\mu \nu}\right|,
$$

where $B^{\alpha \beta}$ denotes the cofactor of the element $b_{\alpha \beta}$ in the determinant $\left|b_{\alpha \beta}\right|$. As so defined the quantities $b^{\alpha \beta}$ are the components of an absolute symmetric contravariant tensor. Then the above equation reduces to one which can be written in the form

$$
\begin{aligned}
& {\left[2\left(H^{2}-K\right) b^{\alpha \beta} H_{\alpha \beta}-4 H b^{\alpha \beta} H_{\alpha} H_{\beta}+2 b^{\alpha \beta} K_{\alpha} H_{\beta}+2 H g^{\alpha \beta} K_{\alpha} H_{\beta}\right.} \\
& \left.\quad-g^{\alpha \beta} K_{\alpha} K_{\beta}-\left(H^{2}-K\right) g^{\alpha \beta} K_{\alpha \beta}-4 K\left(H^{2}-K\right)^{2}\right] b_{12}=0
\end{aligned}
$$

in which for simplicity we have omitted the "commas" denoting covariant differentiation. Again we have

$$
b_{12,1,2}-b_{12,2,1}=b_{11,2,2}-b_{22,1,1}
$$

in view of the Codazzi equations. By Bianchi's identity the left member of this equation becomes $\left(b_{11}-b_{22}\right) K$. The quantities $b_{11,2,2}$ and $b_{22,1,1}$ in the right member can be calculated by covariant differentiation of the third and fourth equations (2.4). When these substitutions are made we obtain an equation which, after considerable reduction, becomes

$$
[]\left(b_{11}-b_{22}\right)=0,
$$

where the bracket stands for the bracket expression in (3.2). 
If $H^{2}-K>0$ it follows from $\S 1$ that either $b_{11}-b_{22} \neq 0$ or $b_{12} \neq 0$. Hence it follows from (3.2) and (3.3) that

$$
\begin{aligned}
& 2\left(H^{2}-K\right) b^{\alpha \beta} H_{\alpha \beta}-4 H b^{\alpha \beta} H_{\alpha} H_{\beta}+2 b^{\alpha \beta} K_{\alpha} H_{\beta}+2 H g^{\alpha \beta} K_{\alpha} H_{\beta} \\
&-g^{\alpha \beta} K_{\alpha} K_{\beta}-\left(H^{2}-K\right) g^{\alpha \beta} K_{\alpha \beta}-4 K\left(H^{2}-K\right)^{2}=0
\end{aligned}
$$

at a point $P$ where $H^{2}-K>0$. From continuity, (3.4) is also valid at a point $P$ where $H^{2}-K=0$ provided that this point $P$ is a limit of points at which $H^{2}-K>0$. Finally suppose that $H^{2}-K=0$ at a point $P$ which is not a limit of points at which $H^{2}-K>0$. Then this point $P$ will be contained in a region $R$ of the surface within which $H^{2}-K=0$. But then the left member of (3.4) is seen directly to vanish at $P$ since $H=$ const. and $K=$ const. in $R$ by the italicized result in $\$ 1$. Hence the equation (3.4) is satisfied at all points of the surface (without regard to whether the quantity $\mathrm{H}_{2}-K$ vanishes or not).

4. Closed surfaces of constant mean curvature. On the basis of the equation (3.4) we can obtain a simple direct proof of the known result that a closed surface (open and compact Riemann space) of constant mean curvature $H$ and non-negative Gaussian curvature $K$ is of constant Gaussian curvature. In fact if $H=$ const., (3.4) becomes

$$
\left(H^{2}-K\right) g^{\alpha \beta} K_{\alpha \beta}+g^{\alpha \beta} K_{\alpha} K_{\beta}+4 K\left(H^{2}-K\right)^{2}=0 .
$$

Integrating the left member of this equation over the surface we find immediately that

$$
\int g^{\alpha \beta} K_{\alpha} K_{\beta} d S+2 \int K\left(H^{2}-K\right)^{2} d S=0 .
$$

If $K \geqq 0$ it follows that each integral must vanish separately. The vanishing of the first integral implies that $K_{\alpha}=0$ or $K=$ const. over the surface.

5. Determination of the quantities $b_{\alpha \beta}$ in general. It is desirable for the requirements of the following calculations to put equation (3.4) into a more contracted form. This can be accomplished by writing

$$
b^{\alpha \beta} Q_{\alpha \beta}=g^{\alpha \beta} P_{\alpha \beta},
$$

where

$Q_{\alpha \beta}=2\left(H^{2}-K\right) H_{\alpha \beta}-4 H H_{\alpha} H_{\beta}+K_{\alpha} H_{\beta}+K_{\beta} H_{\alpha}$,

$P_{\alpha \beta}=\left(H^{2}-K\right) K_{\alpha \beta}+K_{\alpha} K_{\beta}-H K_{\alpha} H_{\beta}-H K_{\beta} H_{\alpha}+2 K\left(H^{2}-K\right)^{2} g_{\alpha \beta}$.

As so defined the $P$ 's and $Q$ 's are the components of symmetric tensors. 
Now consider the equation (5.1) and also the first of the equations (1.1). Write these in the following expanded form

$$
\left\{\begin{array}{l}
Q_{22} b_{11}-2 Q_{12} b_{12}+Q_{11} b_{22}=\left|g_{\mu \nu}\right| g^{\alpha \beta} P_{\alpha \beta}, \\
g_{22} b_{11}-2 g_{12} b_{12}+g_{11} b_{22}=2\left|g_{\mu \nu}\right| H
\end{array}\right.
$$

We use these equations in the determination of the $b_{\alpha \beta}$. The matrix of the coefficient of the $b$ 's in these equations has the same rank as

$$
\left\|\begin{array}{lll}
Q_{22} & Q_{12} & Q_{11} \\
g_{22} & g_{12} & g_{11}
\end{array}\right\|
$$

In general the rank of this matrix will be two. In the calculation which follows we assume this general case and suppose furthermore for definiteness that the last of the second order determinants of the matrix does not vanish. The results obtained will be seen to be independent of the nonvanishing of this particular determinant and will depend in fact only on the assumption that the above matrix has rank two.

Considerable simplification will be obtained by carrying out the calculations at a point $P$ relative to a coordinate system with respect to which $g_{\alpha \beta}=\delta_{\alpha \beta}$ at $P$. The results found under this condition can immediately be put into a general invariant form. The above nonvanishing determinant in the matrix (5.3) now has the value $Q_{12} \neq 0$ and under this condition the equations (5.2) can be solved for $b_{12}$ and $b_{22}$ in terms of $b_{11}$. We have

$$
\begin{aligned}
& b_{12}=\left[\left(Q_{22}-Q_{11}\right) b_{11}+\left(2 H Q_{11}-\sum P_{\alpha \alpha}\right)\right]\left(Q_{12}\right)^{-1 / 2}, \\
& b_{22}=2 H-b_{11} .
\end{aligned}
$$

Now turn to the second of the equations (1.1) and into this equation substitute the above values of $b_{12}$ and $b_{22}$. The result is a quadratic equation in $b_{11}$ the solution of which is

$$
b_{11}=\frac{4 H Q_{12}^{2}-\left(Q_{22}-Q_{11}\right)\left(2 H Q_{11}-\sum P_{\alpha \alpha}\right) \pm A^{1 / 2}}{\left(Q_{22}-Q_{11}\right)^{2}+4 Q_{12}^{2}},
$$

where

$$
\begin{aligned}
A= & {\left[\left(Q_{22}-Q_{11}\right)\left(2 H Q_{11}-\sum P_{\alpha \alpha}\right)-4 H Q_{12}^{2}\right]^{2} } \\
& -\left[\left(Q_{22}-Q_{11}\right)^{2}+4 Q_{12}^{2}\right]\left[\left(2 H Q_{11}-\sum P_{\alpha \alpha}\right)^{2}+4 K Q_{12}^{2}\right] .
\end{aligned}
$$

By multiplying out the factors occurring in the numerator of the expression for $b_{11}$ and combining in an appropriate manner we find that

$$
\begin{aligned}
4 H Q_{12}^{2} & -\left(Q_{22}-Q_{11}\right)\left(2 H Q_{11}-\sum P_{\alpha \alpha}\right) \\
& =\left[\left(\sum P_{\alpha \alpha}\right)\left(\sum Q_{\beta \beta}\right)-4 H\left|Q_{\alpha \beta}\right|\right]+2 Q_{11}\left[H \sum Q_{\alpha \alpha}-\sum P_{\alpha \alpha}\right]
\end{aligned}
$$


and

$$
\begin{aligned}
A=4 Q_{12}^{2}\left[2 H\left(\sum P_{\alpha \alpha}\right)\left(\sum Q_{\beta \beta}\right)-\right. & K\left(\sum Q_{\alpha \alpha}\right)^{2} \\
& \left.-\left(\sum P_{\alpha \alpha}\right)^{2}-4\left(H^{2}-K\right)\left|Q_{\alpha \beta}\right|\right] .
\end{aligned}
$$

Substitution of these values into (5.6) gives

$$
\begin{aligned}
& b_{11}=\frac{\left[\sum P_{\alpha \alpha} \sum Q_{\beta \beta}-4 H\left|Q_{\alpha \beta}\right|\right]}{\left[\left(Q_{22}-Q_{11}\right)^{2}+4 Q_{12}^{2}\right]}+\frac{2\left[H \sum Q_{\alpha \alpha}-\sum P_{\alpha \alpha}\right] Q_{11}}{\left[\left(Q_{22}-Q_{11}\right)^{2}+4 Q_{12}^{2}\right]} \\
& \pm \frac{2 Q_{12}\left[2 H \sum P_{\alpha \alpha} \sum Q_{\beta \beta}-K\left(\sum Q_{\alpha \alpha}\right)^{2}-\left(\sum P_{\alpha \alpha}\right)^{2}-4\left(H^{2}-K\right)\left|Q_{\alpha \beta}\right|\right]^{1 / 2}}{\left[\left(Q_{22}-Q_{11}\right)^{2}+4 Q_{12}^{2}\right]}
\end{aligned}
$$

Now consider the ratio

$$
\frac{2\left[\sum P_{\alpha \alpha} \sum Q_{\beta \beta}-4 H\left|Q_{\alpha \beta}\right|\right]+2\left[H \sum Q_{\alpha \alpha}-\sum P_{\alpha \alpha}\right] \sum Q_{\beta \beta}}{\left[\left(Q_{22}-Q_{11}\right)^{2}+4 Q_{12}^{2}\right]} .
$$

If we expand and rearrange the terms of the numerator and denominator this ratio becomes

$$
\frac{2 H\left(\sum Q_{\alpha \alpha}\right)^{2}-8 H\left|Q_{\alpha \beta}\right|}{\left(\sum Q_{\alpha \alpha}\right)^{2}-4\left|Q_{\alpha \beta}\right|}=2 H
$$

Since $b_{11}+b_{22}=2 H$ we can now immediately deduce the expression for $b_{22}$. We have in fact

$$
b_{22}=\frac{\left[\sum P_{\alpha \alpha} \sum Q_{\beta \beta}-4 H\left|Q_{\alpha \beta}\right|\right]}{\left[\left(Q_{22}-Q_{11}\right)^{2}+4 Q_{12}^{2}\right]}+\frac{2\left[H \sum Q_{\alpha \alpha}-\sum P_{\alpha \alpha}\right] Q_{22}}{\left[\left(Q_{22}-Q_{11}\right)^{2}+4 Q_{12}^{2}\right]}
$$

$$
\mp \frac{2 Q_{12}\left[2 H \sum P_{\alpha \alpha} \sum Q_{\beta \beta}-K\left(\sum Q_{\alpha \alpha}\right)^{2}-\left(\sum P_{\alpha \alpha}\right)^{2}-4\left(H^{2}-K\right)\left|Q_{\alpha \beta}\right|\right]^{1 / 2}}{\left[\left(Q_{22}-Q_{11}\right)^{2}+4 Q_{12}^{2}\right]} \text {. }
$$

To obtain the corresponding expression for $b_{12}$ we substitute the expression for $b_{11}$ given by (5.7) into the right member of (5.4). After cancellation of various terms the resulting equation reduces to

$$
\begin{aligned}
b_{12}= & \frac{2\left(H \sum Q_{\alpha \alpha}-\sum P_{\alpha \alpha}\right) Q_{12}}{\left[\left(Q_{22}-Q_{11}\right)^{2}+4 Q_{12}^{2}\right]} \\
& \pm \frac{\left(Q_{22}-Q_{11}\right)\left[2 H \sum P_{\alpha \alpha} \sum Q_{\beta \beta}-K\left(\sum Q_{\alpha \alpha}\right)^{2}\right.}{\left[\left(Q_{22}-Q_{11}\right)^{2}+4 Q_{12}^{2}\right]}
\end{aligned}
$$

The problem now is to combine equations (5.7), (5.8) and (5.9) 
into a tensor system of recognizable invariantive character. A step in this direction has already been accomplished by the way in which certain terms in the right members of these equations have been written. For example $\sum P_{\alpha \alpha}$ becomes $g^{\alpha \beta} P_{\alpha \beta}$ when referred to arbitrary coordinates. For this purpose we define the following scalars:

$$
\begin{aligned}
W & =\left[\left(Q_{22}-Q_{11}\right)^{2}+4 Q_{12}^{2}\right]=\left(g^{\alpha \beta} Q_{\alpha \beta}\right)^{2}-4\left|Q_{\alpha \beta}\right| /\left|g_{\alpha \beta}\right|, \\
R & =\sum P_{\alpha \alpha} \sum Q_{\beta \beta}-4 H\left|Q_{\alpha \beta}\right|=g^{\alpha \beta} P_{\alpha \beta}{ }^{\mu \nu} Q_{\mu \nu}-4 H\left|Q_{\alpha \beta}\right| /\left|g_{\alpha \beta}\right|, \\
U & =H \sum Q_{\alpha \alpha}-\sum P_{\alpha \alpha}=H g^{\alpha \beta} Q_{\alpha \beta}-g^{\alpha \beta} P_{\alpha \beta},
\end{aligned}
$$

in which the middle members in these equations are the expressions for these scalars when $g_{\alpha \beta}=\delta_{\alpha \beta}$. In terms of these scalars the expression underneath the radical in the above equations can easily be shown to be given by $\left(H^{2}-K\right) W-U^{2}$. Next define the symmetric tensor $V$ with components

$$
V_{\alpha \beta}=\left(Q_{\alpha \mu} \epsilon_{\beta \nu}+Q_{\beta \mu} \epsilon_{\alpha \nu}\right) g^{\mu \nu},
$$

in which the $\epsilon$ 's are the components of the skew-symmetric tensor defined by $\epsilon_{12}=-\epsilon_{21}=\left(\left|g_{\alpha \beta}\right|\right)^{1 / 2}$ and $\epsilon_{11}=\epsilon_{22}=0$. We note in particular that $V_{11}=2 Q_{12}, V_{22}=-2 Q_{12}$ and $V_{12}=Q_{22}-Q_{11}$ when $g_{\alpha \beta}=\delta_{\alpha \beta}$. On the basis of this observation and the above definitions of the scalars $W, R$, and $U$ we now find that equations (5.7), (5.8) and (5.9) can be combined into the system

$$
\text { (5.10) } b_{\alpha \beta}=R W^{-1} g_{\alpha \beta}+2 U W^{-1} Q_{\alpha \beta} \pm\left\{\left[\left(H^{2}-K\right) W-U^{2}\right]^{1 / 2} / W\right\} V_{\alpha \beta} \text {. }
$$

The determination (5.10) of the quantities $b_{\alpha \beta}$ is valid in any region of the surface in which the condition $W>0$ is satisfied. If $W=0$ at a point $P$ we see from the above expression for this scalar that $Q_{11}=Q_{22}$ and $Q_{12}=0$ provided that $g_{\alpha \beta}=\delta_{\alpha \beta}$ at $P$. But then we can write $Q_{\alpha \beta}=\lambda g_{\alpha \beta}$, that is, the quantities $Q_{\alpha \beta}$ are proportional to the quantities $g_{\alpha \beta}$ and hence the matrix (5.3) has rank less than two at $P$. Conversely if this matrix has rank less than two, the $Q$ 's and the $g$ 's are proportional and the scalar $W=0$.

In (5.10) the mean curvature $H$ must be such a function that the conditions

$$
W>0, \quad\left(H^{2}-K\right) W-U^{2} \geqq 0
$$

are satisfied. Conversely if $H$ is any function satisfying the conditions (5.11) it is evident from the derivation of (5.10) that the quantities $b_{\alpha \beta}$ given by (5.10) satisfy the algebraic conditions (1.1).

6. Relations between the invariants. The scalars $W, R$, and $U$ and the tensor $V$ satisfy the following relations 


$$
\begin{gathered}
2 W=g^{\alpha \beta} g^{\mu \nu} V_{\alpha \mu} V_{\beta \nu}, \\
H W=R+g^{\alpha \beta} Q_{\alpha \beta} U .
\end{gathered}
$$

The first of these can be expressed by saying that the scalar $W$ is one-half the square of the tensor $V$. The derivation of these relations can easily be carried out on the basis of the definitions of the invariants $W, R, U$, and $V$ in $\$ 5$.

7. The exceptional case $W=0$. Suppose $W=0$ over a closed surface. Then $Q_{\alpha \beta}=\lambda(x) g_{\alpha \beta}$, and substituting into this equation the value of $Q_{\alpha \beta}$ from $\S 5$ we have

$$
2\left(H^{2}-K\right) H_{\alpha \beta}-4 H H_{\alpha} H_{\beta}+K_{\alpha} H_{\beta}+K_{\beta} H_{\alpha}=\lambda g_{\alpha \beta} .
$$

Now $b^{\alpha \beta} Q_{\alpha \beta}=b^{\alpha \beta} g_{\alpha \beta} \lambda=2 H \lambda$. Hence (5.1) becomes

$$
\begin{aligned}
\left(H^{2}-K\right) g^{\alpha \beta} K_{\alpha \beta}+g^{\alpha \beta} K_{\alpha} K_{\beta}-2 H g^{\alpha \beta} & K_{\alpha} H_{\beta} \\
& +4 K\left(H^{2}-K\right)^{2}=2 H \lambda .
\end{aligned}
$$

Next put $H^{2}-K=\theta(\geqq 0)$ or $K=H^{2}-\theta$, from which we deduce

$$
\begin{aligned}
K_{\alpha} & =2 H H_{\alpha}-\theta_{\alpha}, \\
K_{\alpha \beta} & =2 H_{\alpha} H_{\beta}+2 H H_{\alpha \beta}-\theta_{\alpha \beta} .
\end{aligned}
$$

When these substitutions are made in (7.2) and the quantities $H_{\alpha \beta}$ in the resulting equations are eliminated by means of (7.1), we have an equation which reduces to

$$
2 \theta g^{\alpha \beta} H_{\alpha} H_{\beta}-\theta g^{\alpha \beta} \theta_{\alpha \beta}+g^{\alpha \beta} \theta_{\alpha} \theta_{\beta}+4 K \theta^{2}=0 .
$$

Now integrate the left member of (7.3) over the closed surface. We thus find that

$$
\int \theta g^{\alpha \beta} H_{\alpha} H_{\beta} d S+\int g^{\alpha \beta} \theta_{\alpha} \theta_{\beta} d S+2 \int K \theta^{2} d S=0 .
$$

If $K>0$ at every point, each of the integrands is non-negative and hence must vanish over the surface. Hence from the third integral we must have $\theta=0$ and from $\S 1$ this means that the mean curvature and Gaussian curvature are constant. Hence if the scalar $W$ vanishes over a closed surface of positive Gaussian curvature, the mean curvature $H$ and the Gaussian curvature $K$ are constant over the surface.

It follows from the above result that for the case of a closed analytic surface which is of positive but not of constant Gaussian curvature the scalar $W$ cannot vanish over any region $R$ of the surface. Hence $W$ can vanish only at exceptional points and elsewhere on the 
surface the determination of the second fundamental form given by (5.10) will apply.

8. The differential equations satisfied by the mean curvature. We have observed ( $\$ 5)$ that the quantities given by (5.10) satisfy (1.1) provided that $H$ is a function satisfying (5.11). However in order for the function $H$ to be the mean curvature of a surface and have the above quantities $b_{\alpha \beta}$ as the components of its second fundamental form the Codazzi equations must also be satisfied, that is, we must have $b_{\alpha \beta, \gamma}=b_{\alpha \gamma, \beta}$. It may be observed that for the case of the twodimensional surface under consideration these latter equations can be written in the contracted form

$$
b_{\alpha \beta, \gamma} \epsilon^{\beta \gamma}=0 .
$$

Understanding that the $b_{\alpha \beta}$ are given by (5.10) in terms of the mean curvature $H$ and its derivatives, we see that the two equations (8.1) are the differential equations satisfied by the mean curvature $H$. Conversely if the scalar function $H$ satisfies the conditions (5.11) and the differential equations (8.1) over a given abstract two-dimensional Riemann space, then the quantities $b_{\alpha \beta}$ defined by (5.10) together with the function $H$ will be the components of a possible second fundamental form and the mean curvature respectively for the given Riemann space considered as a surface in three-dimensional Euclidean space.

INDIANA UNIVERSITY 